\title{
Effect of Psychosocial Factors on Cancer Risk and Survival
}

\author{
Naoki Nakaya
}

Division of Personalized Prevention and Epidemiology, Department of Preventive Medicine and Epidemiology,

Tohoku Medical Megabank Organization, Tohoku University, Sendai, Japan

Received August 30, 2013; accepted October 15, 2013; released online November 23, 2013

Copyright (c) 2013 Naoki Nakaya. This is an open access article distributed under the terms of Creative Commons Attribution License, which permits unrestricted use, distribution, and reproduction in any medium, provided the original author and source are credited.

\begin{abstract}
Psychosocial factors such as personality traits and depression may alter immune and endocrine function, with possible effects on cancer incidence and survival. Although these factors have been extensively studied as risk and prognostic factors for cancer, the associations remain unclear. The author used data from prospective cohort studies in population-based and clinical databases to investigate these relations. The findings do not support the hypotheses that personality traits and depression are direct risk factors for cancer and cancer survival.

Some researchers have recently reported that cancer affects the psychological status of the partners and family members of cancer patients. The mechanisms underlying this hypothesis imply the existence of not only psychological distress from caregiving and grief but also a shared unhealthy lifestyle. Only a few studies have suggested that major psychosocial problems develop in partners of cancer patients. The present study used nationwide population-based data to investigate depression risk among male partners of women with breast cancer. The results support the hypothesis that such men are at increased risk of depression.

In conclusion, the effects of personality traits and depression on cancer risk and survival appear to be extremely small. In addition, partners of cancer patients were at increased risk of depression. Screening partners and family members of cancer patients for depressive symptoms is therefore an important concern for research in psychooncology.
\end{abstract}

Key words: cancer; cohort study; depression; personality; psychosocial factors; risk; survival

\section{PERSONALITY TRAITS AND CANCER RISK}

Personality traits have long been hypothesized to have a causal role in cancer development and progression. In 1962, Kissen and Eysenck conducted one of the first modern studies on the association between personality traits and cancer and reported that, as compared with hospital controls, patients with lung cancer were more likely to be extraverted and less likely to be neurotic, ${ }^{1}$ which could be interpreted to indicate that extraverts are at increased risk of cancer because they seek stimulation and thus experience high levels of stress, whereas individuals with low levels of neuroticism could be at increased risk of cancer because they tend to have fewer emotional outlets and therefore accumulate emotional stress. ${ }^{2}$ Greater exposure to stress could affect cancer risk by influencing immune and endocrine function. , $^{3,4}$

Since then, several well-conducted prospective studies found no association between personality traits (eg, extraversion, neuroticism, and trait anxiety) and cancer risk. However, most of these studies had methodologic limitations, including small numbers of incident cancers, which limited the statistical power to analyze site-specific cancer.

The present study used data from 2 large prospective cohort studies (the Miyagi cohort study ${ }^{5}$ and Swedish twin cohort/ Finnish twin cohort $^{6}$ ) to investigate the association between personality traits and cancer risk.

From June through August 1990, 30277 residents of Miyagi Prefecture, in northern Japan, completed a Japanese version of the short form of the Eysenck Personality Questionnaire-Revised (EPQ-R) and a questionnaire on health habits. ${ }^{5}$ There were 671 prevalent cases of cancer at baseline, and 986 incident cases of cancer were identified during the 7-year follow-up, through December 1997. Multivariable hazard ratios (HRs) for total cancer among individuals in the highest versus the lowest quartile for a personality trait subscale were 0.9 for extraversion $(95 \%$ $\mathrm{CI}=0.7-1.1 ; \quad P$ for linear trend $=0.32)$ and 1.2 for 
Table 1. Multivariate-adjusted hazard ratios (HRs) and $95 \%$ Cls for cancer risk according to score quartile of personality trait subscale

\begin{tabular}{|c|c|c|c|c|c|c|c|c|c|}
\hline \multirow[b]{2}{*}{ Miyagi cohort study ${ }^{5}$} & \multirow{2}{*}{$\begin{array}{l}\text { EPQ-R subscales } \\
\text { Score group }\end{array}$} & \multicolumn{4}{|c|}{ EPQ-R, Extraversion } & \multicolumn{4}{|c|}{ EPQ-R, Neuroticism } \\
\hline & & Q1 (low) & Q2 & Q3 & Q4 (high) & Q1 (low) & Q2 & Q3 & Q4 (high) \\
\hline \multirow{4}{*}{$\begin{array}{l}\text { Outcome: } \\
\text { Total cancer } \\
\text { incidence }\end{array}$} & $\begin{array}{l}\text { Multivariate HR } \\
(95 \% \mathrm{Cl})\end{array}$ & $\begin{array}{l}1.0 \\
\text { (Ref) }\end{array}$ & $\begin{array}{c}0.8 \\
(0.7-1.0)\end{array}$ & $\begin{array}{c}0.9 \\
(0.8-1.1)\end{array}$ & $\begin{array}{c}0.9 \\
(0.7-1.1)\end{array}$ & $\begin{array}{l}1.0 \\
\text { (Ref) }\end{array}$ & $\begin{array}{c}1.0 \\
(0.8-1.2)\end{array}$ & $\begin{array}{c}1.0 \\
(0.9-1.2)\end{array}$ & $\begin{array}{c}1.2 \\
(1.0-1.4)\end{array}$ \\
\hline & $P$ for linear trend & \multicolumn{4}{|c|}{0.32} & \multicolumn{4}{|c|}{0.06} \\
\hline & Covariates & \multicolumn{8}{|c|}{ Age, sex, smoking, alcohol, body mass index, education, family history of cancer } \\
\hline & EPI subscales & \multicolumn{4}{|c|}{ EPI, Extraversion } & \multicolumn{4}{|c|}{ EPI, Neuroticism } \\
\hline $\begin{array}{l}\text { Swedish/Finnish } \\
\text { twin cohort }{ }^{6}\end{array}$ & Score group & \multicolumn{4}{|c|}{ Continuous variables } & \multicolumn{4}{|c|}{ Continuous variables } \\
\hline \multirow{3}{*}{$\begin{array}{l}\text { Outcome: } \\
\text { Total cancer } \\
\text { incidence }\end{array}$} & $\begin{array}{l}\text { Multivariate HR } \\
(95 \% \mathrm{Cl})\end{array}$ & \multicolumn{4}{|c|}{$\begin{array}{c}0.99 \\
(0.98-1.01)\end{array}$} & \multicolumn{4}{|c|}{$\begin{array}{c}1.00 \\
(0.99-1.02)\end{array}$} \\
\hline & $P$ for linear trend & \multicolumn{4}{|c|}{0.23} & \multicolumn{4}{|c|}{0.48} \\
\hline & Covariates & \multicolumn{8}{|c|}{ Age, sex, smoking, alcohol, body mass index, education } \\
\hline
\end{tabular}

Abbreviations: EPG-R, Eysenck Personality Questionnaire-Revised; EPI, Eysenck Personality Inventory; Q, quartile.

Scores on personality subscales were divided into 4 categories to yield quartiles.

neuroticism $(95 \% \mathrm{CI}=1.0-1.4 ; P$ for linear trend $=0.06)$ (Table 1)

Additional analyses examined how study design (retrospective or prospective) and duration of follow-up (in prospective analyses) affected associations between personality scales and risk of total cancer. In the retrospective analysis, the 671 cancer cases at baseline, which had been ascertained from self-reports in the healthhabit questionnaire or from cancer registry records, were used as an endpoint. Unconditional logistic regression was used to estimate odds ratios for the presence of prevalent cancers in relation to quartile of personality subscale scores. In the prospective analyses, 2 durations of follow-up were used: the first analysis encompassed the first 3 years of follow-up after the baseline, and the second encompassed 7 years of followup but excluded cancer cases diagnosed within the first 3 years. In analyses that examined the effect of study design (ie, retrospective vs prospective) and duration of follow-up (in the prospective study) on associations between personality subscales and risk of total cancer, the association with neuroticism differed depending on the type of analysis performed (Figure). Retrospective analysis (OR) showed a significant positive linear association between neuroticism and presence of cancer at baseline $(P$ for linear trend $<0.001)$. Prospective analysis with only 3 years of follow-up (HR1) showed a significant positive linear association between neuroticism and the HR for incident cancer $(P$ for linear trend $=0.03$ ). However, in the second prospective analysis, which considered individuals during a follow-up period of 7 years but excluded cancer cases diagnosed in the first 3 years of follow-up (HR2), neuroticism was not associated with risk of incident cancer $(P$ for linear trend $=0.43$ ). These findings do not support the hypothesis that personality traits are a risk factor for cancer incidence. The association between neuroticism and prevalent cancer may be a consequence rather than a cause of cancer diagnosis and symptoms. ${ }^{5}$

The second prospective population-based cohort study comprised 59548 Swedish (1974-1999) and Finnish (1976-2004) participants, who completed a questionnaire that included the Eysenck Personality Inventory (EPI) and items on health behavior at baseline. ${ }^{6}$ To analyze the association of extraversion and neuroticism with cancer risk, 4631 cancer cases were identified during a maximum followup period of 30 years. The present author used Cox proportional hazards models to estimate HRs for incidence of any cancer. HRs were estimated by treating personality trait subscale scores as continuous variables and are presented as risk per 1-unit increase in score for each scale. In multivariate analyses, extraversion and neuroticism were not significantly associated with overall cancer risk: the HRs were 0.99 for extraversion $(95 \% \mathrm{CI}=0.9-1.01 ; P$ for linear trend $=0.23)$ and 1.00 for neuroticism $(95 \% \mathrm{CI}=0.99-1.02 ; P$ for linear trend $=0.48)$ (Table 1). To the author's knowledge, this is the largest study (>4500 incident cases) of the associations between personality traits and cancer risk. The findings are in line with those of recent prospective studies, which do not support the hypothesis that personality traits are direct risk factors for overall cancer risk.

\section{PERSONALITY TRAITS AND SURVIVAL AFTER CANCER}

It as been suggested that personality traits have a role in cancer progression. Temoshok et al observed that tumor thickness in patients with malignant melanoma was positively associated with a "type C" personality, which they described as cooperative, unassertive, patient, suppressive of negative emotions, and accepting/compliant with external authorities. ${ }^{7}$ 


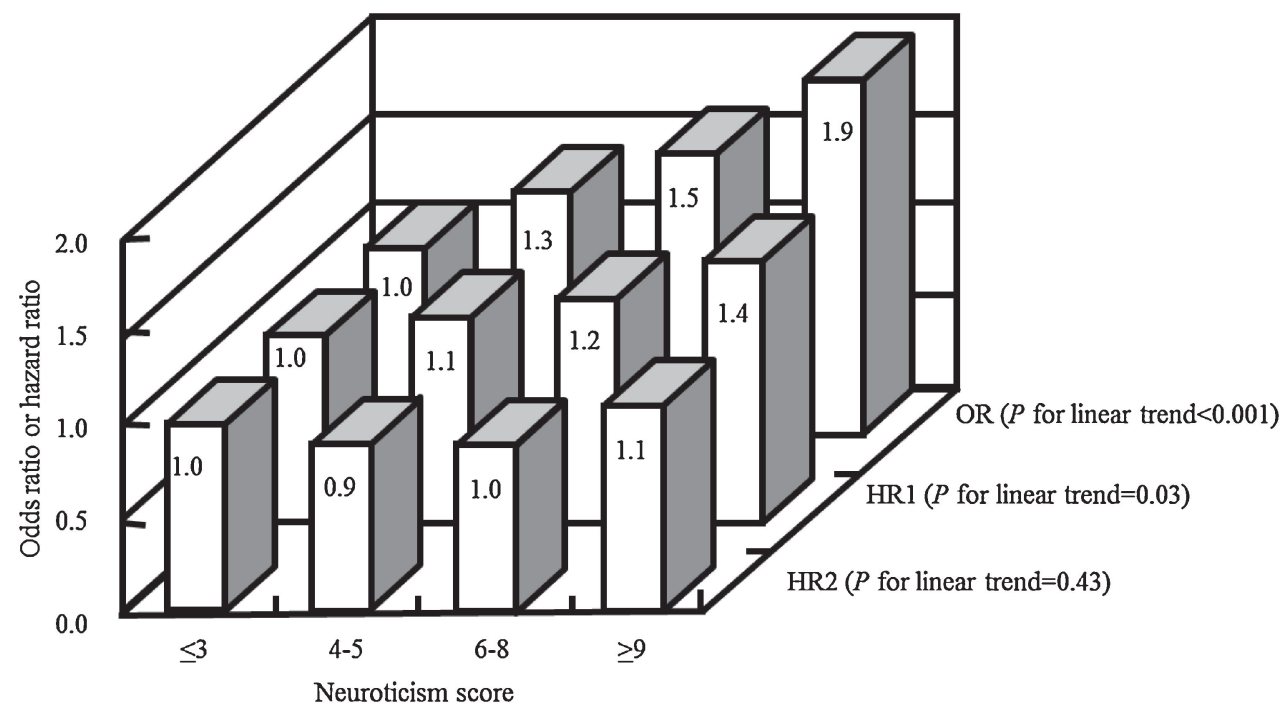

Figure. Association between neuroticism and overall cancer risk, according to study design (retrospective or prospective) and duration of follow-up (in prospective analyses). ${ }^{5}$ Cut-off points for neuroticism score were chosen so as to divide the population into 4 groups of similar size. In all analyses, the referent group was the group with the lowest neuroticism scores. OR denotes an odds ratio estimated from retrospective analysis of 671 prevalent cases of cancer at the baseline as the endpoint $(P$ for linear trend $<0.001)$. HR1 denotes a hazard ratio estimated from prospective analysis of 320 incident cases of cancer diagnosed in the first 3 years of follow-up as the endpoint $(P$ for linear trend $=0.03$ ). HR2 denotes a hazard ratio estimated from prospective analysis of 666 incident cases of cancer diagnosed in years 4-7 of follow-up -ie, excluding cancer cases diagnosed in the first 3 years of followup-as the endpoint $(P$ for linear trend $=0.43$ ). All ORs and HRs were adjusted for sex, age, cigarette smoking (never smoker, past smoker, currently smoking 1-19 cigarettes per day, or currently smoking $\geq 20$ cigarettes per day), alcohol consumption (never drinker, past drinker, currently drinking $\leq 22.7 \mathrm{~g}$ of alcohol per day, or currently drinking $\geq 22.8 \mathrm{~g}$ of alcohol per day), body mass index ( $\leq 18.4,18.5-24.9$, or $\geq 25.0 \mathrm{~kg} / \mathrm{m}^{2}$ ), education (in school until age 15 years, $16-18$ years, or $\geq 19$ years), and family history of cancer (presence or absence in first-degree relatives).

Patients with low extraversion and high neuroticism are believed to repress their emotions, which is considered one of the most important aspects of the type $\mathrm{C}$ personality. ${ }^{8}$ The hypothesis regarding cancer survival could also be interpreted as being related to stress. Accumulated repression of emotions may cause stress, which could affect cancer progression by influencing immune and endocrine function.,

The role of personality traits in survival after cancer has been addressed in several prospective studies, but the evidence is limited and no conclusion has been reached. These studies had several limitations, such as small sample size. Almost all had fewer than 200 participants and lacked sufficient statistical power to analyze site-specific cancer. The present author used 2 population-based prospective cohort studies (the Miyagi cohort study ${ }^{9}$ and Finnish twin cohort $^{6}$ ) to test the hypothesis that personality traits have a role in cancer survival.

First, in July 1990, 41442 residents of Japan completed the short form of the EPQ-R and a questionnaire on various health habits; 890 incident cases of cancer were identified among the participants between January 1993 and December 1997. ${ }^{9}$ These 890 cases were followed-up until March 2001, and 356 deaths from all causes were identified among them. Cox proportional hazards models were used to estimate HRs for death according to score quartiles for the 4 personality trait subscales, after adjustment for potential confounders. Multivariable HRs for all-cause death among individuals in the highest versus the lowest quartile of personality trait subscale score were 1.0 for extraversion $(95 \% \mathrm{CI}=0.8-1.4$; $P$ for linear trend $=0.73)$ and 1.1 for neuroticism $(95 \%$ $\mathrm{CI}=0.8-1.6 ; P$ for linear trend $=0.24)($ Table 2$)$.

The second large prospective population-based cohort study comprised 31145 Finnish (baseline investigation period= 1976-2004) participants who had completed a questionnaire including the EPI and items on health behavior at baseline. ${ }^{6} \mathrm{~A}$ total of 2733 cancer cases were identified during a maximum follow-up period of 30 years, among whom there were 1548 deaths during a maximum follow-up period of 29 years. Cox proportional hazards models were used to estimate HRs for all-cause death. HRs were estimated by treating scores on personality traits subscales as continuous variables and are presented as risk per 1-unit increase in score for each scale. In multivariate analyses, extraversion and neuroticism were not significantly associated with all-cause death: the HRs were 1.00 for extraversion $(95 \% \mathrm{CI}=0.98-1.02 ; P$ for linear trend $=0.86)$ and 1.00 for neuroticism $(95 \% \mathrm{CI}=0.98-1.02$; $P$ for linear trend $=0.61)$ (Table 2 ).

The data from these Japanese and Finnish population-based prospective cohort studies do not support the hypothesis that personality traits are associated with cancer survival. 
Table 2. Multivariate-adjusted hazard ratios (HRs) and $95 \%$ Cls for all-cause mortality according to score quartile for personality traits subscales among persons with a diagnosis of cancer

\begin{tabular}{|c|c|c|c|c|c|c|c|c|c|}
\hline \multirow[b]{2}{*}{ Miyagi cohort study ${ }^{9}$} & \multirow{2}{*}{$\frac{\text { Exposure }}{\text { Score group }}$} & \multicolumn{4}{|c|}{ EPQ-R, Extraversion } & \multicolumn{4}{|c|}{ EPQ-R, Neuroticism } \\
\hline & & Q1 (low) & Q2 & Q3 & Q4 (high) & Q1 (low) & Q2 & Q3 & Q4 (high) \\
\hline \multirow{4}{*}{$\begin{array}{l}\text { Outcome: } \\
\text { Mortality after } \\
\text { cancer diagnosis }\end{array}$} & $\begin{array}{l}\text { Multivariate HR } \\
(95 \% \mathrm{Cl})\end{array}$ & $\begin{array}{l}1.0 \\
\text { (Ref) }\end{array}$ & $\begin{array}{c}0.8 \\
(0.7-1.0)\end{array}$ & $\begin{array}{c}0.9 \\
(0.8-1.1)\end{array}$ & $\begin{array}{c}0.9 \\
(0.7-1.1)\end{array}$ & $\begin{array}{l}1.0 \\
\text { (Ref) }\end{array}$ & $\begin{array}{c}1.0 \\
(0.8-1.2)\end{array}$ & $\begin{array}{c}1.0 \\
(0.9-1.2)\end{array}$ & $\begin{array}{c}1.2 \\
(1.0-1.4)\end{array}$ \\
\hline & $P$ for linear trend & \multicolumn{4}{|c|}{0.32} & \multicolumn{4}{|c|}{0.06} \\
\hline & Covariates & \multicolumn{8}{|c|}{ Age, sex, smoking, alcohol, body mass index, education, family history of cancer } \\
\hline & EPI subscales & \multicolumn{4}{|c|}{ EPI, Extraversion } & \multicolumn{4}{|c|}{ EPI, Neuroticism } \\
\hline Swedish/Finnish & Score group & \multicolumn{4}{|c|}{ Continuous variables } & \multicolumn{4}{|c|}{ Continuous variables } \\
\hline \multirow{3}{*}{$\begin{array}{l}\text { Outcome: } \\
\text { Mortality after } \\
\text { cancer diagnosis }\end{array}$} & $\begin{array}{l}\text { Multivariate HR } \\
(95 \% \mathrm{Cl})\end{array}$ & \multicolumn{4}{|c|}{$1.00(0.98-1.02)$} & \multicolumn{4}{|c|}{$1.00(0.98-1.02)$} \\
\hline & $P$ for linear trend & \multicolumn{4}{|c|}{0.86} & \multicolumn{4}{|c|}{0.61} \\
\hline & Covariates & \multicolumn{8}{|c|}{ Age, sex, smoking, alcohol, body mass index, education } \\
\hline
\end{tabular}

Abbreviations: EPG-R, Eysenck Personality Questionnaire-Revised; EPI, Eysenck Personality Inventory; Q, quartile.

Scores on personality subscales were divided into 4 categories to yield quartiles.

\section{DEPRESSION AND SURVIVAL AFTER CANCER}

Negative psychological states, including depression, are common among cancer patients. Between $8 \%{ }^{10}$ and $44 \%{ }^{11}$ of patients with lung cancer were reported to have depression. Furthermore, it has been suggested that depression affects prognosis and quality of life among patients with lung cancer. In a recent review of studies on associations between depression and mortality risk among cancer patients, depression was associated with mortality risk. ${ }^{12}$ It has been hypothesized that depression affects mortality risk in cancer patients through endocrine and/or immunologic pathways ${ }^{13,14}$ or through poor compliance with cancer treatment. ${ }^{15}$ Another possible explanation for the increased mortality observed among cancer patients with depression is that depression may simply reflect poor clinical status, which by itself would be associated with increased cancer mortality. Depression was reported to be strongly associated with poor clinical status, as indicated by tumor stage, performance status (PS), and severity of clinical symptoms. In addition, severity of clinical symptoms such as pain and dyspnea was an important independent prognostic factor in a population that included patients with lung cancer. Indices of clinical status are thus important confounders when evaluating the association between depression and cancer mortality. The present study tested the hypothesis that the association between depression and cancer survival among patients with lung cancer is confounded by the poor clinical status of patients. ${ }^{16}$

The author conducted a prospective cohort study using data from the Lung Cancer Database Project at the National Cancer Center Hospital East in Japan. ${ }^{16,17}$ Between July 1999 and July 2004, 1178 patients with lung cancer were enrolled. The questionnaire included items on socioeconomic characteristics, smoking status, clinical symptoms, and psychological status after diagnosis. Depression status among patients with lung cancer was assessed by using the Hospital Anxiety and Depression Scale. Information on clinical stage, PS, and histological type was obtained from medical charts. The participants were followed-up until December 2004, and 686 died. A Cox regression model was used to estimate HRs for all-cause death. Adjustment for the effects of socioeconomic variables and smoking status (model 1) and for the effects of clinical stage and PS as indicators of clinical status (model 2) did not alter the significant positive association between depression and mortality ( $P$ for linear trend $<0.001$ and 0.04 , respectively). However, when selfreported pain and dyspnea were included in the multivariate model (model 3), the association became nonsignificant $(P$ for linear trend $=0.26)$ (Table 3$)$. Multivariate logistic regression analysis using a cross-sectional design to examine the association between indicators of clinical status and depression among study patients showed that more-advanced clinical stage and poorer PS were significantly associated with higher prevalence of depression. Further, more-severe pain and dyspnea were significantly associated with higher prevalence of depression, independent of clinical stage or PS.

The present findings indicate that the association between depression and mortality risk among patients with lung cancer was largely confounded by indicators of clinical status, including clinical stage, PS, and clinical symptoms. ${ }^{16}$

\section{RISK OF DEPRESSION AMONG PARTNERS OF CANCER PATIENTS}

Understanding of the psychosocial consequences of cancer has increased during the past few decades. In response, supportive psychosocial intervention strategies have been 
Table 3. Multivariate-adjusted hazard ratios (HRs) and $95 \%$ Cls for all-cause mortality according to score quartile for depression subscale among patients with a diagnosis of lung cancer

\begin{tabular}{|c|c|c|c|c|c|c|c|c|c|c|c|c|c|}
\hline \multirow{3}{*}{$\begin{array}{l}\text { Lung Cancer } \\
\text { Database } \\
\text { Project, } \\
\text { Japan }^{16}\end{array}$} & Exposure & \multicolumn{12}{|c|}{ HADS, Depression } \\
\hline & Score group & Q1 (low) & Q2 & Q3 & Q4 (high) & Q1 (low) & Q2 & Q3 & Q4 (high) & Q1 (low) & Q2 & Q3 & Q4 (high) \\
\hline & $\begin{array}{l}\text { Multivariate HR } \\
(95 \% \mathrm{Cl}) \\
\end{array}$ & $\begin{array}{c}1.0 \\
\text { (Ref) }\end{array}$ & $\begin{array}{c}1.3 \\
(1.0-1.6)\end{array}$ & $\begin{array}{c}1.4 \\
(1.1-1.7)\end{array}$ & $\begin{array}{c}1.8 \\
(1.5-2.3) \\
\end{array}$ & $\begin{array}{c}1.0 \\
\text { (Ref) }\end{array}$ & $\begin{array}{c}1.0 \\
(0.8-1.2) \\
\end{array}$ & $\begin{array}{c}1.0 \\
(0.8-1.3)\end{array}$ & $\begin{array}{c}1.3 \\
(1.0-1.6)\end{array}$ & $\begin{array}{c}1.0 \\
\text { (Ref) }\end{array}$ & $\begin{array}{c}1.0 \\
(0.8-1.2)\end{array}$ & $\begin{array}{c}1.0 \\
(0.8-1.3)\end{array}$ & $\begin{array}{c}1.2 \\
(0.9-1.4)\end{array}$ \\
\hline \multirow[b]{2}{*}{$\begin{array}{l}\text { Outcome: } \\
\text { Mortality after } \\
\text { cancer } \\
\text { diagnosis }\end{array}$} & $P$ for linear trend & \multicolumn{4}{|c|}{$<0.001$} & \multicolumn{4}{|c|}{0.04} & \multicolumn{4}{|c|}{0.26} \\
\hline & Covariates & \multicolumn{4}{|c|}{$\begin{array}{l}\text { Model 1: } \\
\text { Age at diagnosis, sex, histologic type, } \\
\text { education, marital status, smoking }\end{array}$} & \multicolumn{4}{|c|}{$\begin{array}{l}\text { Model 2: } \\
\text { Age at diagnosis, sex, histologic type, } \\
\text { education, marital status, smoking, } \\
\text { clinical status, PS }\end{array}$} & \multicolumn{4}{|c|}{$\begin{array}{l}\text { Model 3: } \\
\text { Age at diagnosis, sex, histologic type, } \\
\text { education, marital status, smoking, } \\
\text { clinical status, PS, } \\
\text { self-reported pain, dyspnea }\end{array}$} \\
\hline
\end{tabular}

Abbreviations: HADS, Hospital Anxiety and Depression Scale; PS, performance status, Q, quartile.

Scores on personality subscales were divided into 4 categories to yield quartiles.

Table 4. Multivariate-adjusted hazard ratios (HRs) and $95 \%$ Cls for affective disorder among partners of a woman with breast cancer

\begin{tabular}{llc}
\hline $\begin{array}{l}\text { Nationwide study, } \\
\text { Denmark }\end{array}$ & Exposure & Breast cancer diagnosis in partner \\
\cline { 2 - 3 } & Multivariate HR $(95 \% \mathrm{Cl})$ & $1.39(1.20-1.61)$ \\
\cline { 2 - 3 } $\begin{array}{l}\text { Outcome: } \\
\text { Hospitalization for an } \\
\text { affective disorder }\end{array}$ & Covariates & $\begin{array}{l}\text { Number of children, highest attained educational level, disposable household income, } \\
\text { affiliation with labor market, Charlson index, history of alcohol-related mental disorders }\end{array}$ \\
\cline { 2 - 2 }
\end{tabular}

developed and are tailored to the problems that cancer patients face during the course of their disease. The extent to which cancer affects patients and their closest relatives was first addressed in a seminal article published more than 20 years ago. House et al illustrated how several diseases affected people close to the patient. ${ }^{18}$ The mechanisms of these effects may involve several interacting pathways: the event may cause stress in the partner; it may deprive the partner of emotional, social, and economic support; and it can influence the daily life and behavior of the partner. ${ }^{18,19}$ The effect of cancer on the psychological well-being of partners could increase the risk of several psychiatric disorders related to stressful life events, including neurotic, stress-related, somatoform, substance abuse-related, and affective disorders.

The few small studies that have been published thus far suggest that serious psychosocial problems develop among partners of cancer patients; however, to the best of the author's knowledge, no studies have addressed the risk of severe depression.

A retrospective cohort study of male partners of women with breast cancer used unbiased nationwide population-based data to investigate their risk of hospitalization for an affective disorder. ${ }^{20}$ The study followed 1162596 men born during 1925-1973 who were older than 30 years at the time of the study, resided in Denmark between 1994 and 2006, had no history of hospitalization for an affective disorder, and had continuously lived with the same partner for at least 5 years. The Cox regression analysis included detailed clinical information on the diagnosis and treatment of breast cancer and on annually updated socioeconomic and health-related data obtained from national administrative and disease registers. During the 13-year follow-up period, breast cancer was diagnosed in the partners of 20538 men. In multivariable analysis, men whose partner was diagnosed with breast cancer were at increased risk of being hospitalized with an affective disorder $\quad(\mathrm{HR}=1.39, \quad 95 \% \quad \mathrm{CI}=1.20-1.61, \quad P<0.001$; Table 4), and there was a dose-response relation between breast cancer severity and risk of hospitalization. Furthermore, the risk of hospitalization for an affective disorder among men whose partner had died after a breast cancer diagnosis was 3.6-fold that of men whose partner had survived breast cancer.

\section{CONCLUSIONS AND FUTURE IMPLICATIONS}

\section{Personality traits and cancer risk}

The association between neuroticism and prevalent cancer may be a consequence rather than a cause of cancer diagnosis and symptoms. Further, although residual confounding may never be totally eliminated, the present findings strongly suggest that the overall effect size for a causal association between personality and cancer is extremely small. Thus, such an association, if it exists at all, is unlikely to have clinical or public health implications.

\section{Personality traits and survival after cancer}

Personality traits such as extraversion and neuroticism are not direct risk factors for survival after cancer. 


\section{Depression and survival after cancer}

The association between depression and mortality risk among patients with lung cancer was largely confounded by indicators of clinical status, including clinical stage, PS, and clinical symptoms.

\section{Risk of depression among partners of cancer patients}

Men whose partner had breast cancer were at increased risk of hospitalization for an affective disorder. Screening for depressive symptoms among partners and family members of cancer patients is therefore an important issue for research in psycho-oncology.

\section{ONLINE ONLY MATERIALS}

Abstract in Japanese.

\section{ACKNOWLEDGMENTS}

The author would like to thank Professor Emeritus Shigeru Hisamichi (Tohoku University) and Professors Ichiro Tsuji (Tohoku University), Shin Fukudo (Tohoku University), Shinichi Kuriyama (Tohoku University), Atsushi Hozawa (Tohoku University), and Yoshitaka Tsubono (Yamagata Sakuracho Hospital) for guiding me in the academic discipline of epidemiology. I would also like to thank to Professors Yosuke Uchitomi (Okayama University) and Christoffer Johansen (Danish Cancer Society, Denmark) for guiding me in the field of psycho-oncology. I am also obliged to all the study participants for giving me the opportunity to perform this series of studies, and to all staff who have supported me. This study was supported by a Grant-in-Aid from the Japan Society for the Promotion of Science (Kakenhi 23650420; Japan) and by the Foundation for Promotion of Cancer Research (Japan) for the 3rd Term Comprehensive 10-year Strategy for Control (Japan).

Conflicts of interest: None declared.

\section{REFERENCES}

1. Kissen DM, Eysenck HJ. Personality in male lung cancer patients. J Psychosom Res. 1962;6:123-7.

2. Eysenck HJ. Personality, stress and cancer: prediction and prophylaxis. Br J Med Psychol. 1988;61(Pt 1):57-75.

3. Kiecolt-Glaser JK, Glaser R. Psychoneuroimmunology and cancer: fact or fiction? Eur J Cancer. 1999;35(11):1603-7.

4. Antoni MH, Lutgendorf SK, Cole SW, Dhabhar FS, Sephton SE, McDonald PG, et al. The influence of bio-behavioural factors on tumour biology: pathways and mechanisms. Nat Rev Cancer.
2006;6(3):240-8.

5. Nakaya N, Tsubono Y, Hosokawa T, Nishino Y, Ohkubo T, Hozawa A, et al. Personality and the risk of cancer. J Natl Cancer Inst. 2003;95:799-805.

6. Nakaya N, Bidstrup PE, Saito-Nakaya K, Frederiksen K, Koskenvuo M, Pukkala E, et al. Personality traits and cancer risk and survival based on Finnish and Swedish registry data. Am J Epidemiol. 2010;172(4):377-85.

7. Temoshok L, Heller BW, Sagebiel RW, Blois MS, Sweet DM, DiClemente RJ, et al. The relationship of psychosocial factors to prognostic indicators in cutaneous malignant melanoma. J Psychosom Res. 1985;29(2):139-53.

8. Eysenck HJ. The prediction of death from cancer by means of personality/stress questionnaire: too good to be true? Percept Mot Skills. 1990;71(1):216-8.

9. Nakaya N, Tsubono Y, Nishino Y, Hosokawa T, Fukudo S, Shibuya D, et al. Personality and cancer survival: the Miyagi cohort study. Br J Cancer. 2005;92:2089-94.

10. Uchitomi Y, Mikami I, Nagai K, Nishiwaki Y, Akechi T, Okamura H. Depression and psychological distress in patients during the year curative resection of non-small-cell lung cancer. J Clin Oncol. 2003;21:69-77.

11. Montazeri A, Milroy R, Hole D, McEwen J, Gillis CR. Anxiety and depression in patients with lung cancer before and after diagnosis: findings from a population in Glasgow, Scotland. J Epidemiol Community Health. 1998;52:203-4.

12. Satin JR, Linden W, Phillips MJ. Depression as a predictor of disease progression and mortality in cancer patients: a metaanalysis. Cancer. 2009;115(22):5349-61.

13. Levy S, Herberman R, Lippman M, d'Angero T. Correlation of stress factors with sustained depression of natural killer cell activity and predicted prognosis in patients with breast cancer. J Clin Oncol. 1987;5:348-53.

14. Pettingale KW, Philalithis A, Tee DE, Greer HS. The biological correlates of psychological responses to breast cancer. J Psychosom Res. 1981;25:453-8.

15. Colleoni M, Mandala M, Peruzzotti G, Robertson C, Bredart A, Goldhirsch A. Depression and degree of acceptance of adjuvant cytotoxic drugs. Lancet. 2000;356:1326-7.

16. Nakaya N, Saito-Nakaya K, Akechi T, Kuriyama S, Inagaki M, Kikuchi N, et al. Negative psychological aspects and survival in lung cancer patients. Psychooncology. 2008;17:466-73.

17. Nakaya N, Goto K, Saito-Nakaya K, Inagaki M, Otani T, Akechi $\mathrm{T}$, et al. The lung cancer database project at the National Cancer Center, Japan: study design, corresponding rate and profiles of cohort. Jpn J Clin Oncol. 2006;36(5):280-4.

18. House JS, Landis KR, Umberson D. Social relationships and health. Science. 1988;241:540-5.

19. Berkman LF, Kawachi I. Social Epidemiology. New York: Oxford University Press; 2000.

20. Nakaya N, Saito-Nakaya K, Bidstrup PE, Dalton SO, Frederiksen K, Steding-Jessen $\mathrm{M}$, et al. Increased risk of severe depression in male partners of women with breast cancer. Cancer. 2010;116:5527-34. 